NASA/TM-2003-212264

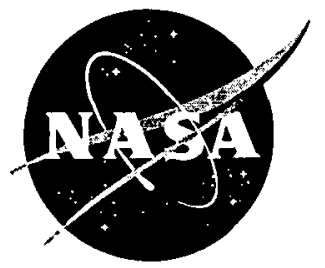

\title{
The Effect of Symbology Location and Format on Attentional Deployment within a Cockpit Display of Traffic Information
}

Walter W. Johnson

Ames Research Center, Moffett Field, California

Min-Ju Liao

National Research Council

Stephen Tse

San Jose State University Foundation, San Jose, California

National Aeronautics and

Space Administration

Ames Research Center

Moffett Field, California 94035

April 2003 
Available from:

NASA Center for AeroSpace Information 7121 Standard Drive

Hanover, MD 21076-1320

301-621-0390
National Technical Information Service 5285 Port Royal Road Springfield, VA 22161 703-605-6000

This report is also available in electronic form at http://human-factors.arc.nasa.gov/ihh/cdti/index.html 


\section{Summary}

The present experiment employed target detection tasks to investigate attentional deployment during visual search for target aircraft symbols on a cockpit display of traffic information (CDTI). Targets were defined by either a geometric property (aircraft on a collision course with Ownship) or a textual property (aircraft with associated altitude tags indicating an even altitude level). Effects of target location and target brightness (highlighting) were examined. Target location was systematically related to target detection time, and this interacted with the target's defining property (collision geometry or associated text). Highlighting (which was not linked to whether an aircraft symbol was the target) did not influence target detection time.

\section{Introduction}

The design of informationally dense cockpit displays of traffic information (CDTIs) poses significant challenges to display designers. High among these challenges is ensuring that pilots pay sufficient attention to the most important information, while minimizing the time needed to scan the CDTI. In order to achieve these aims, research is needed at both theoretical and applied levels. At a theoretical level, Egeth and Yantis (1997) have identified three central issues determining selection/control in visual attention: the topdown (goal-directed) or bottom-up (stimulusdriven) nature of attentional control; spatially oriented or object-oriented basis of attentional selection; and the time course of attention. At a more applied level, designers need more information concerning how pilots tend to spatially deploy their attention over these displays, and also on how display elements may be modified to increase (or decrease) their effective salience (the ability to attract attention). This study addresses these issues by measuring the effect of display location on attention, and also by examining the effect of brightness highlighting on attention.

\section{Display Location}

Researchers have been debating the role of location in attentional deployment, and how attention moves or shifts between locations. For example, one issue is whether the time needed to shift attention from one location to another in the visual field is distancedependent or distance-independent. Studies by Sagi and Julesz (1985), Kwak, Dagenbach, and Egeth (1991), and Sperling and Weichselgartner (1995) have suggested that relocation of attention is independent of distance. However, Shulman, Remington, and McLean (1979) and Tsal (1983) obtained evidence that attention moves in an analog fashion and thus moving attention over greater distances requires more time. Another issue is whether there are inherent biases to attend to different display locations. Wolfe, O'Neil, and Bennett (1998) and Previc and Blume (1993) investigated this using visual search tasks and found that participants responded to upper and right visual fields more quickly than lower and left visual fields, respectively. Irrespective of the discrete/analog distinction, there is a need to determine the temporal course of information pick-up from a CDTI in order to discover biases in attending to different regions on the display and also in order to more effectively capitalize on, or remediate, these biases.

\section{Effective Salience}

One method of modifying the order of information processing in multi-element displays is the use of highlighting.

Highlighting is the use of specific stimulus attributes (e.g., brightness, color, blinking) to attract attention.

Highlighting has been studied at both the theoretical and the applied level. For example, some researchers have conducted theoretical studies of top-down vs. bottom-up attentional control. Folk and Remington (1998) used a 
modified spatial cueing paradigm and showed that attention is contingent on top-down "control settings" such as the defining feature of the target. Gibson \& Jiang (1998), in their unexpected color singleton experiments, also reported that attentional control was not driven by stimuli. Jonides and Yantis (1998) similarly showed that color and brightness singletons did not capture attention. However, some studies have suggested that items with salient features will be processed first and therefore bottom-up processing also plays an important role in visual search tasks (e.g., Joseph \& Optican, 1996; Kawahara \& Toshima, 1997). For example, Pashler (1988) in his experiment asked participants to search for a slash $(/)$ in an array of many Os or for an $\mathrm{O}$ among slashes. Although the colors of items were irrelevant to the task, they were manipulated in the experiment. Results showed that the reaction time to locate the target shape was longer when the color singletons appeared, despite participants' intention to ignore them. Theeuwes (1991a, 1992) has found in his experiments that singletons that were irrelevant to the task could also capture participants' attention.

More applied studies have examined the use of highlighting to direct users' initial attention to a target/targets on a display with the goal of reducing search time (e.g., Morse, 1979; Smith \& Goodwin, 1971, 1972; Stewart, 1976). However, highlighting is not always beneficial. Fisher and Tan (1989) found that the type of highlighting, the level of highlighting validity, and the probability that users attend first to the highlighted options all determine whether highlighting produced performance profits. Of greatest interest in the present study are the findings on highlighting validity which show that highlighting, that is non-valid (nonpredictive), has no effect on search. This contrasts with the findings from some of the theoretical studies, cited above, which have shown bottom-up effects, but is consistent with other studies and theories which give a greater role to top-down control.
The goals of the present experiment were twofold. The first goal was to obtain a spatialtemporal description of the deployment of attention during visual search tasks on a CDTI. Second, this study has examined the bottomup effect of stimulus salience (brightness highlighting) on this attentional deployment.

\section{Method}

\section{Stimuli and Design}

The experiment employed a CDTI upon which nine aircraft symbols, and one Ownship symbol were displayed (Figure 1). Ownship was indicated by a filled triangular symbol near the bottom of the display. All symbols were chevrons, and the directions of all aircraft were indicated by the orientation of these chevrons. In addition, each aircraft symbol had an associated altitude tag. Two target detection tasks (collision detection and altitude detection), and one collision evaluation task, were employed.

In both detection tasks, the participants were required to search for a single target aircraft among 8 distractor aircraft. In the Altitude Detection Task, targets were defined by a textual property - whether the altitude tag associated with an aircraft indicated an even numbered flight level (e.g. 360, 380, 400). In the Altitude Detection Task the distractor aircraft all occupied odd numbered flight levels.

In the Collision Detection Task, targets were defined by a geometric property - whether an aircraft was on a collision course with Ownship (participants were told to assume that all aircraft flew at the same speed). In the Collision Detection Task all distractor aircraft were designed to miss Ownship by a visually large margin. This was done in order to make the difference between targets and distractors immediate and obvious, and thereby to minimize the time needed to evaluate a particular aircraft. This was required since the 
time needed to evaluate a potential collision may very well be a function of the specific geometry for that collision. Since the goal of the study was to evaluate the time needed to detect a target as a function of location and highlighting, it was important to minimize differences in response time due to collision evaluation differences at various locations.

Finally, the Collision Evaluation Task was included in order to verify that evaluation time differences at various locations did not account for the findings on the Collision Detection Task. In each trial of this task a circle was initially displayed at some location on the display, thus directing the participant's attention to this location. This circle was then replaced by an aircraft symbol, and the participant was required to evaluate if it was on a collision course with Ownship. In this manner, the search component of the response time was eliminated, and the dependence of evaluation time on aircraft location could be assessed.

Three factorial designs were created and examined in this study. The first two designs (described below) presented nine aircraft symbols ( 1 target and 8 distractors) per trial, and examined performance on the two target detection tasks. The first design was created by a factorial crossing of $\mathrm{X}$ region location (left, middle, right), Y region location (bottom, middle, top), Task (Altitude Detection and Collision Detection), and Symbol Brightness (bright, dim). The $X$ and $Y$ target regions were created by partitioning the cockpit display (see Figure 1) into nine equally sized $x-y$ regions (by trisecting the display width and height into three equal parts). By examining participants' response times for targets from different $x-y$ regions, the impact of location on visual search could be tested and the spatio-temporal pattern (if any) of the visual search could be revealed. Additionally, whether this spatio-temporal pattern depended on the nature of the task could be assessed. Finally, whether the brightness of the targets made them inherently easier to search could be assessed (it was hoped that the dim and bright targets would be equally easy to search).

The second design used the same manipulations of $\mathrm{X}$ and $\mathrm{Y}$ target regions and of Task. However brightness was manipulated in a different fashion. For this design every trial presented an approximately equal mixture of bright and dim aircraft symbols, but target brightness was manipulated. On half of the trials the target aircraft symbol was bright, and on half it was dim (thus highlighting had a zero correlation with target status, and thus was not a valid predictor). The goal of the brightness manipulation was to determine if non-predictive highlighting would benefit search performance above and beyond any intrinsic benefit revealed in the analysis of the first design.

Finally, the third design was used to examine the Collision Evaluation Task in which only one aircraft was presented. Again the aircraft appeared equally often in the nine $x-y$ regions, and again the symbols were equally often bright and dim. There was no Task factor, but there was a Threat factor, since the presented

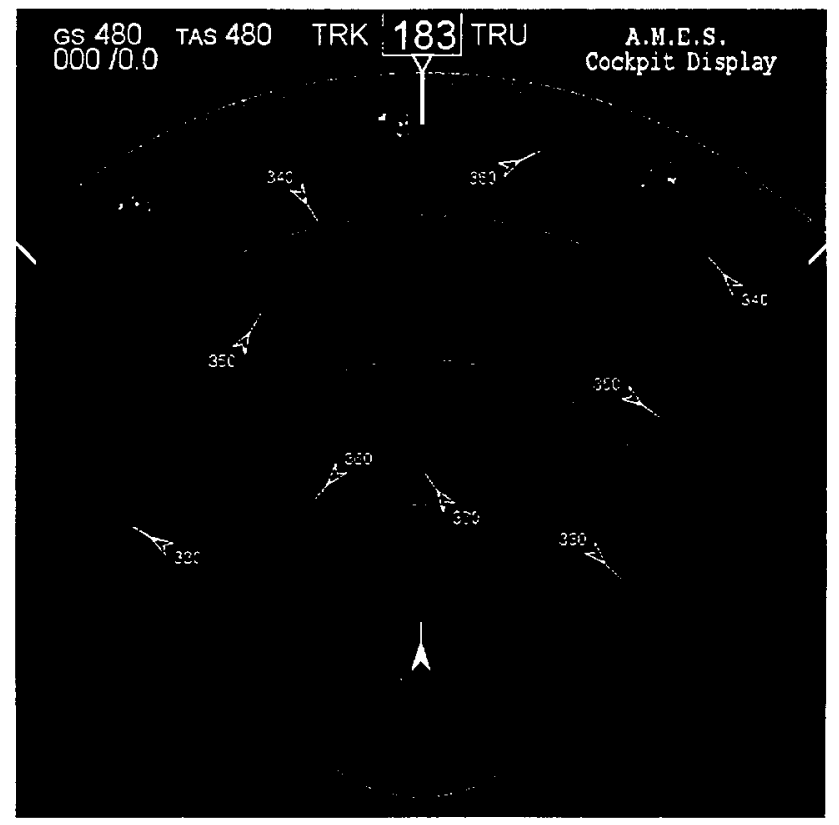

Figure 1. Cockpit Display of Traffic Information. 
aircraft was equally often on a collision and non-collision course. The only purpose of this task was to test for any (unwanted) effects of target location on the target evaluation response time.

\section{Participants}

Eight university students participated in the present experiment. All were financially compensated for their time.

\section{Apparatus}

An Intergraph Pentium 200 system with a 19inch diagonal SVGA (1024 x 1280) display was used for this study. Viewing distance was approximately two feet, and the display updated 60 times per second.

\section{Procedure}

Participants were given detailed instructions and then an experimenter reviewed critical aspects of the tasks and procedures. The three tasks were ordered such that Altitude Detection Task was always presented second. Half of the subjects received the Collision Detection Task first, and the Collision Evaluation Task last, while the remaining participants received the reverse order. Participants received 10 practice trials before each task. For all tasks, participants were told to make an assessment as quickly as possible without sacrificing accuracy. Auditory feedback was provided informing participants whether they were correct or incorrect. Accuracy and response time were measured. The response time was defined from the time the aircraft appeared on the display until the time participants responded.

\section{Results}

\section{Analyses of Variance}

The average error rates for the Collision Evaluation Task, the Collision Detection Task, and the Altitude Detection Task were low: $3.4 \%, 2.94 \%$, and $1.96 \%$, respectively.
Furthermore, there was no discernable relationship between these errors and response times. For each participant, trials with response times more than 4 standard deviations from average response time were considered as outliers and omitted from further analyses. Overall, about $1 \%$ of the trials was excluded from each task.

Two within-subject ANOVAs were used to analyze the target detection data. One of the two ANOVAs used data from trials in which the highlighting was uniform (i.e., all targets were either not highlighted, or all targets were highlighted). The second ANOVA used data from trials which presented mixed highlighting (i.e., half of the aircraft were highlighted and half of the aircraft were not highlighted). The factors used in both analyses were: Task (Collision Detection and Altitude Detection); $\mathrm{X}$ and $\mathrm{Y}$ target location; and Target Brightness. The results showed that response time was not influenced by target brightness in either ANOVA. However, in both analyses response time was systematically related to $x-y$ target location. Furthermore, the nature of this $x-y$ relationship depended upon the task (tests of the three-way Task $x \mathrm{X}$ Position $x$ Y-Position interactions yielded $\mathrm{F}(4,28)=5.477, \mathrm{p}<.01$, for mixed highlighting, and $F(4,28)=5.411, \mathrm{p}<.01$, for uniform highlighting). Figure 2 shows the pattern of response times across the nine regions for the two tasks (these data are the average of the mixed and uniform highlighting data). It can be seen that different attentional deployment patterns were associated with the different tasks. Participants appeared to locate target aircraft most rapidly when they were near the center of the display, with detection speed dropping as targets were located more toward the periphery. However, it can also be seen that this pattern is not truly circular, but more horseshoes shaped, with detection times remaining high at the bottom of the display close to Ownship. 

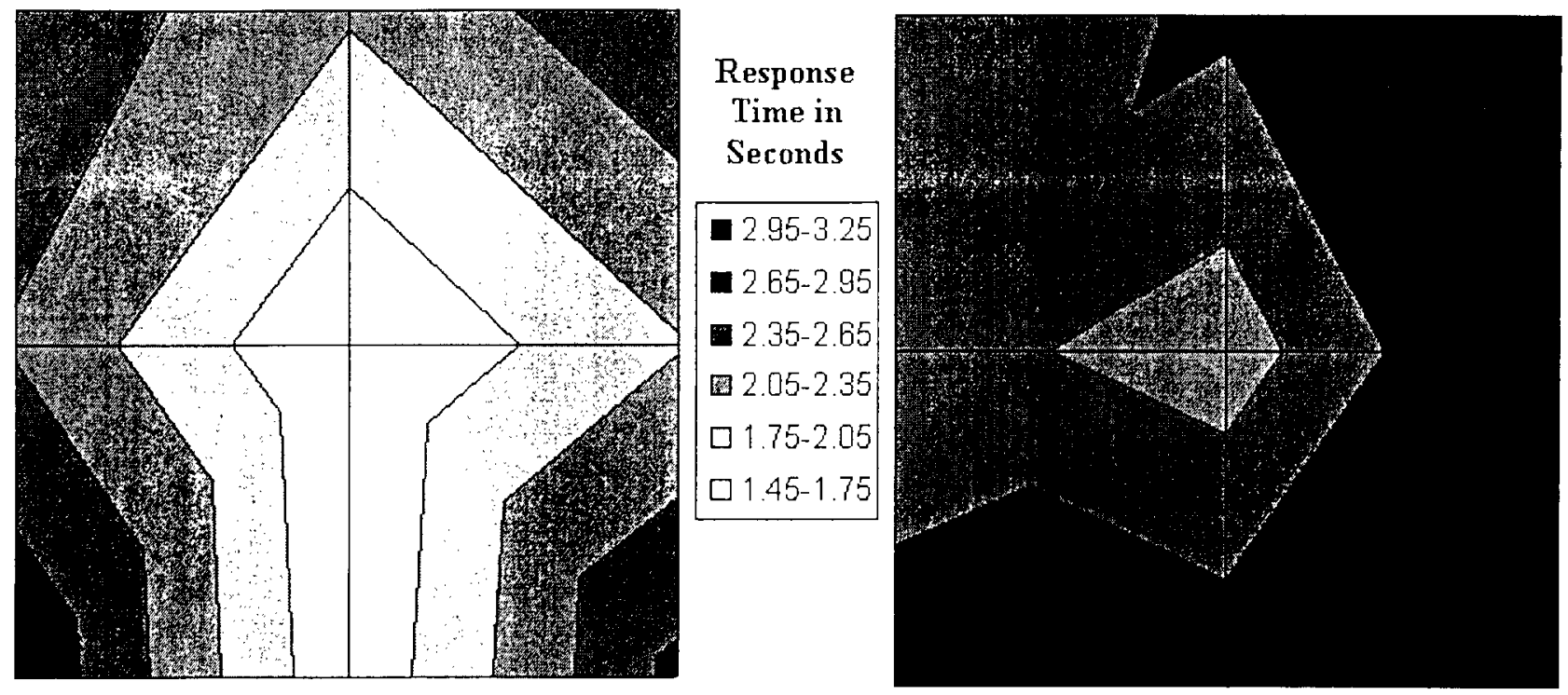

Figure 2. Reaction time (search plus evaluation) as function of display location in nine alternative conflict detection task (left panel) and altitude detection task (right panel).

The pattern is remarkably different for the Altitude Detection Task. Here participants appeared to locate target aircraft most rapidly when they were near the center of the display, and otherwise tended to detect targets more rapidly on the left of the display.

Finally, Figure 3 shows the results of the Collision Evaluation Task. Here there was a significant effect of location in the $\mathrm{X}$ (lateral) display dimension $(\mathrm{F}(2,14)=33.6, \mathrm{p}<.001)$. However, this effect is very small (averaging less than $100 \mathrm{msec}$ difference between the central regions and the peripheral regions) compared to the variation in response time for the Collision Detection Task, which generated region dependent differences more than ten times that magnitude. This difference in effect size, together with the different pattern, strongly suggests that the findings in the Collision Detection Task are due to spatial biases in allocation of attention, and not to differences in evaluation difficulty associated with different locations.

\section{Regression Analyses}

To further understand how participants deployed their attention on the display, the data were fit with linear regression models.
These models predicted response time using the distances from an initial search position $\left(\mathrm{x}_{0}, \mathrm{y}_{\mathrm{o}}\right)$, which were also estimated parameters in these regression models. The cockpit display range was from -42.5 to $42.5 \mathrm{NM}$ along the lateral $\mathrm{X}$-axis and from -20 to 80 NM along the vertical Y-axis, with Ownship located at $(0,0)$. In addition, the absolute value of the target bearing with respect to Ownship was used as a predictor variable in the Collision Detection Task. The fits to the Collision Detection Task and the Altitude Detection Task yielded correlations of 0.75 and 0.59 , respectively. The best fitting $\left(x_{10}, y_{0}\right)$ for the two tasks indicate that participants initiated their search for the colliding target toward the lower center of the display $\left(\left(x_{0}, y_{0}\right)\right.$ $=(1.17,27.77))$, while initiating their search more toward the left of the display for the altitude target $\left(\left(x_{0}, y_{0}\right)=(-16.36,43.34)\right)$. In order to further suppress noise variability, these models were then used to predict the average response times for each of the 9 regions. The result of this was to raise the correlations to 0.96 for the Collision Detection Task and 0.94 for the Altitude Detection Task.

\section{Discussion}




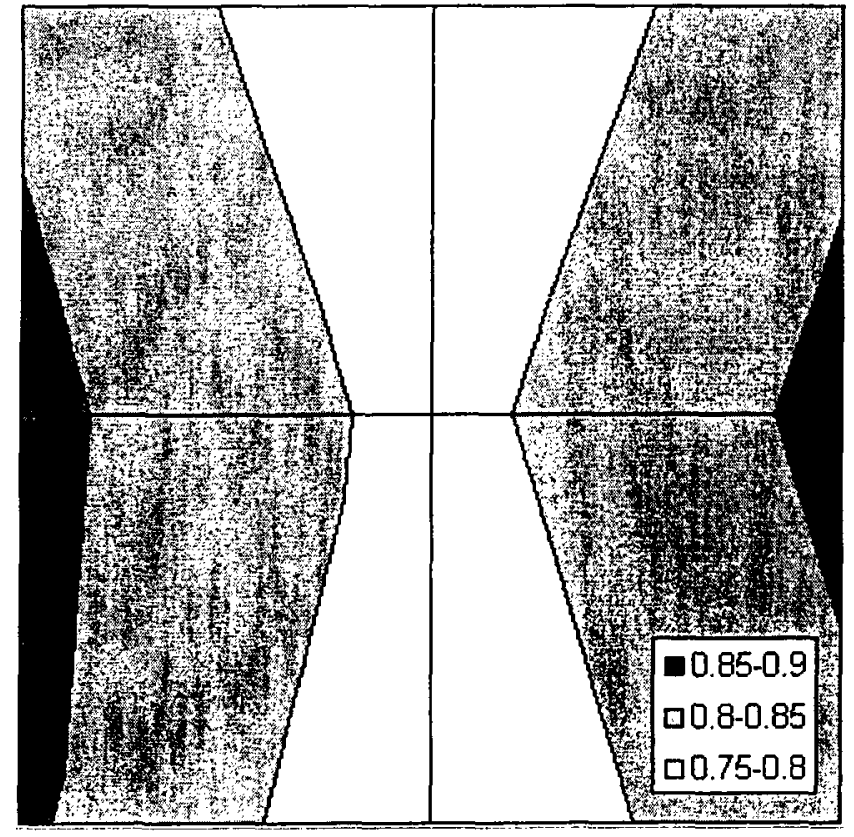

Figure 3. Reaction (evaluation) time as function of display location in single alternative conflict evaluation task.

Although the present data did not show superior performance over the upper and right visual fields as was reported by Wolfe et al. (1998) and Previc and Blume (1993), the present data did reveal that participants had biases to attend to different display locations in a visual search task. And the attentional biases appeared to be different for different tasks. It therefore suggests that both the inherent bias and task property play important roles in the attentional deployment. The bias revealed in the Collision Detection Task is similar to that found in a previous study by Delzell, Johnson, \& Liao (1998). That study tested retention of CDTI aircraft information by airline pilots whom had been instructed to use the CDTI to evaluate their ability to maneuver Ownship within the traffic situation. That study found similar circular patterns, but with Ownship at the center. The left-right bias in the Altitude Detection Task also suggests that a natural left to right reading tendency may be influencing performance.

On the other hand, brightness appeared to have no effect on attracting participants' initial attention. This may imply that the attentional control in the present experiment was more of a top-down (goal-directed) process. However, the present experiment did not test other types of highlighting such as blinking and color. Also, the effects of highlighting may be affected by the proportion of highlighted items, and by how they cluster, on the display. Future examinations of bottom-up effects of highlighting should focus on testing different types of highlighting, and upon how the number of proportion of highlighted items affects attention.

In summary, the present experiment investigated attentional deployment during visual search for target aircraft symbols on a CDTI. Of particular interest in the present experiment were a spatial-temporal description of attentional deployment in a visual search task and the effect of stimulus salience on this attentional deployment. Effects of target location and target brightness were examined. The results showed that simple highlighting of targets did not influence target detection time when that highlighting was randomly related to whether or not an aircraft symbol was a target. However, target location was systematically related to target detection time, and this interacted with the nature of the detection task.

\section{References}

Delzell, S., Johnson, W., \& Liao, M. (1998). Pilots' spatial mental models for memory of heading and altitude. Proceedings of the $17^{\text {th }}$ Digital Avionics Systems Conference, Bellevue, Washington.

Egeth, H. E. \& Yantis, S. (1997). Visual attention: control, representation, and time course. Annual Review Psychology, 48, 26997.

Fisher, D. L. \& Tan, K. C. (1989). Visual displays: The highlighting paradox. Human Factors, 31(1), 17-30. 
Folk, C. L. \& Remington, R. (1998).

Selectivity in distraction by irrelevant featural singletons: Evidence for two forms of attentional capture. Journal of Experimental Psychology: Human Perception and Performance, 24(3), 847-848.

Gibson, B. S. \& Jiang, Y. (1998). Surprise! An unexpected color singleton does not capture attention in visual search. Psychological Science, 9(3), 176-182.

Jonides, J. \& Yantis, S. (1988). Uniqueness of abrupt visual onset in capturing attention. Perception and Psychophysics, 43, 346-354.

Joseph, J. S. \& Optican L. M. (1996). Involuntary attentional shifts due to orientation differences. Perception and Psychophysics, 58, 651-665.

Kee, D., Jung, E. S., \& Chung, M. K. (1992). Isoresponse time regions for the evaluation of visual search performance in ergonomic interface models. Ergonomics, 35(3), 243252.

Kawahara, J. \& \& Toshima, T. (1997). Stimulus-driven control of attention: evidence from visual search for moving target among static nontargets. Japanese Journal of Psychonomic Science, Mar, 15(2), 77-87.

Morse, A. (1979). Some principles for the effective display of data. Computer Graphics, 13, 94-101.

Pashler, H. (1988). Cross -dimensional interaction and texture segregation. Perception and Psychophysics, 43, 307-318.

Podgorny, P. \& Shepard, R. N. (1983). Distribution of visual attention over space. Journal of Experimental Psychology: Human Perception and Performance, 9(3), 380-393.

Previc, F. H., Blume, J. L. (1993). Visual search asymmetries in three-dimensional space. Vision Research, 33(18), 2697-2704.
Sagi, D. \& Julesz, B. (1985). Fast noninertial shifts of attention. Spatial Vision, 2, 141-149.

Shulman, G. L., Remington, R. W., McLean, J. P. (1979). Moving attention through visual space. Journal of Experimental Psychology: Human Perception and Performance, 5, 522526.

Smith, S. L. \& Goodwin, N. C. (1971). Blink coding for information display. Human Factors, 13, 283-290.

Smith, S. L., \& Goodwin, N. C. (1972). Another look at blinking displays. Human Factors, 14, 345-347.

Stewart, T. F. M. (1976). Displays and the software interface. Applied Ergonomics, 7, 137-146.

Theeuwes, J. (1991a). Cross-dimensional perceptual selectivity. Perception and Psychophysics, 50, 184-193.

Tsal, Y. (1983). Movement of attention across the visual field. Journal of Experimental Psychology: Human Perception and Performance, 9, 523-530.

Wolfe, J. M., O’Neil, P., \& Bennett, S. C. (1998). Why are there eccentricity effects in visual search? Visual and attentional hypotheses. Perception \& Psychophysics, 60(1), 140-156. 


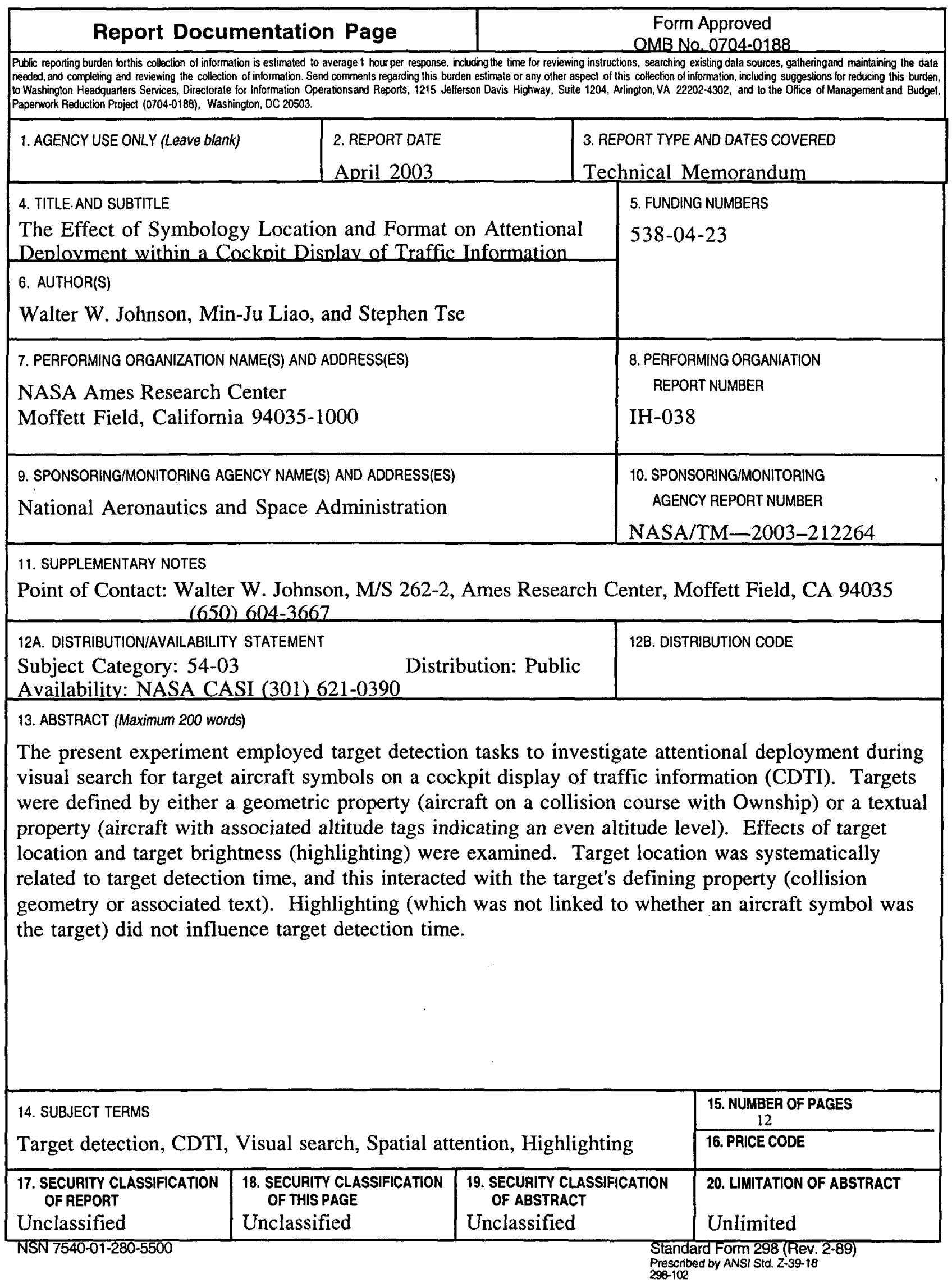

